EPJ Web of Conferences 41, 10009 (2013)

DOI: $10.1051 /$ epjconf/20134110009

(C) Owned by the authors, published by EDP Sciences, 2013

\title{
Ultrafast thin disk lasers: sub-100 fs pulse duration and carrier envelope offset detection
}

\author{
Clara J. Saraceno ${ }^{1}$, Selina Pekarek ${ }^{1}$, Oliver H. Heckl ${ }^{1}$, Cyrill R. E. Baer ${ }^{1}$, Cinia Schriber ${ }^{1}$, \\ Matthias Golling ${ }^{1}$, Kolja Beil ${ }^{2}$, Christian Kränkel ${ }^{2}$, Günter Huber ${ }^{2}$, Thomas Südmeyer ${ }^{1,3}$ and \\ Ursula Keller ${ }^{1}$ \\ ${ }^{1}$ Physics Department, Institute for Quantum Electronics, ETH Zurich, Switzerland \\ ${ }^{2}$ Institute of Laser-Physics, University of Hamburg, Germany \\ ${ }^{3}$ Department of Physics, Time and Frequency Laboratory, University of Neuchâtel, Switzerland
}

\begin{abstract}
We present two milestone results that confirm that thin disk lasers are excellent candidates for driving extreme nonlinear optics experiments. In a first experiment using the broadband mixed sesquioxide gain material $\mathrm{Yb}: \mathrm{LuScO}_{3}$, we demonstrate that thin disk lasers can access the sub-100 fs regime. In a second experiment, we detect for the first time the carrier envelope offset (CEO) frequency beat of an $\mathrm{Yb}: \mathrm{Lu}_{2} \mathrm{O}_{3}$ thin disk laser.
\end{abstract}

\section{Introduction}

SESAM modelocked thin disk lasers (TDLs) [1,2] currently achieve the highest average powers and pulse energies of any femtosecond oscillator technology. Average powers $>140 \mathrm{~W}[3,4]$ and pulse energies $>40 \mu \mathrm{J}[4]$ have been demonstrated directly from such oscillators. Very recently, the highest average power demonstrated was increased to $>270 \mathrm{~W}$ with a pulse duration of $538 \mathrm{fs}$. This power scalable technology results in ideal table-top sources for experiments that require high peak powers and benefit from $\mathrm{MHz}$ repetition rates such as high harmonic generation [5]. The resulting compact coherent UV sources find applications in a broad range of fields such as precision spectroscopy, metrology and attosecond science.

Driving these highly nonlinear processes efficiently requires short pulse durations $\left(\tau_{\mathrm{p}}<100 \mathrm{fs}\right)$. Currently, record high power TDLs operate at pulse durations $>500 \mathrm{fs}$ and require external pulse compression schemes to reach the sub-100 fs regime (Fig. 1a). Obtaining shorter pulses directly from such sources is a topic of extensive investigation [6]. One of the main challenges is finding materials that combine excellent spectroscopic and thermo-mechanical properties suitable to manufacture thin disks with the required optical quality. Furthermore, stabilized sources are crucial in most of the targeted applications and the carrier envelope offset (CEO) properties of modelocked TDLs had, up-to-date, never been investigated. 

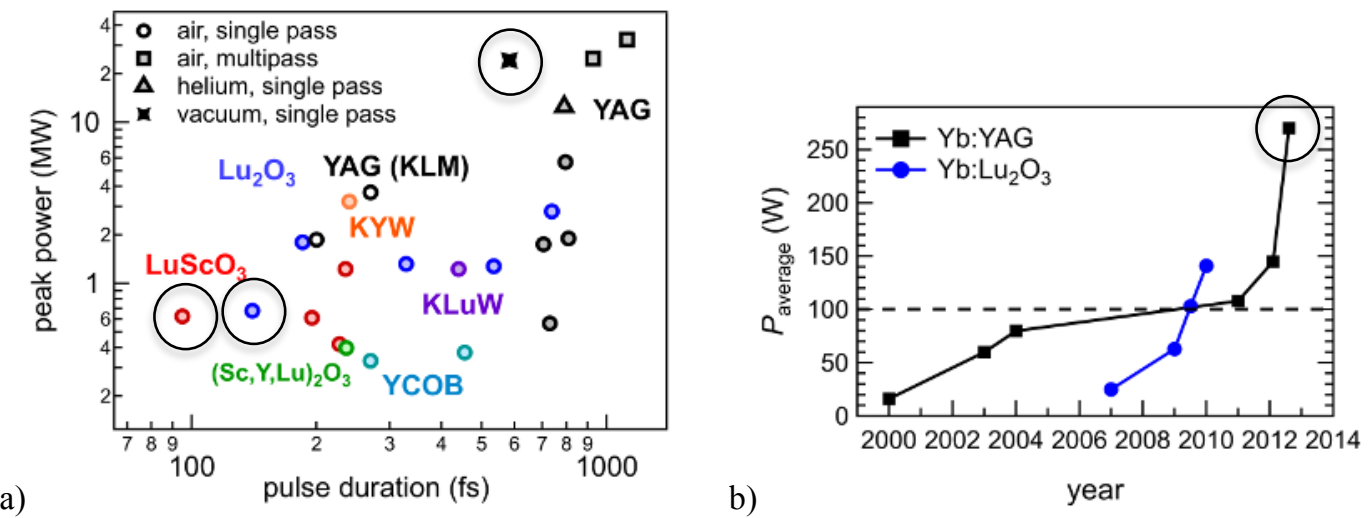

Fig. 1. a) Peak power of modelocked TDLs versus their pulse duration. Highlighted: the first sub-100 fs $\mathrm{Yb}: \mathrm{LuScO}_{3} \mathrm{TDL}$, the $\mathrm{Yb}: \mathrm{Lu}_{2} \mathrm{O}_{3}$ thin disk laser used for the first measurement of the CEO frequency and the most recent average power scaling result obtained using Yb:YAG b) Evolution of average power of modelocked thin disk lasers since their first demonstration in the year 2000.

In this paper, we demonstrate that TDLs can access the sub-100 fs regime directly and we present the first measurement of the CEO frequency of a modelocked TDL, two important milestones that confirm the suitability of modelocked TDLs as driving sources for high field physics experiments.

\section{Results}

The sub-100 fs TDL was based on the broadband mixed sesquioxide gain material $\mathrm{Yb}: \mathrm{LuScO}_{3}$ (22 nm full-width half maximum (FWHM) emission bandwidth). A schematic of the laser setup is presented in Fig. 2a. The details of the different components can be found in Ref [7]. Stable modelocking was obtained with up to $5.1 \mathrm{~W}$ of average power at a pulse duration of $96 \mathrm{fs}$ (Fig. 2b) and a repetition rate of $77.5 \mathrm{MHz}$, with an optical-to-optical efficiency of $11 \%$. These are, to our knowledge, the shortest pulses ever obtained with a modelocked TDL, reaching for the first time the sub-100 fs regime. Although the average output power obtained in this proof of principle experiment is moderate, making use of the high intracavity peak power $(\sim 25 \mathrm{MW})$ of such a source for initial experiments is already an interesting possibility. Further power scaling will be achieved by using larger disks, larger beams on both the SESAM and the gain medium, and by designing SESAMs with optimized parameters for high power operation and short pulse generation [8].

a)

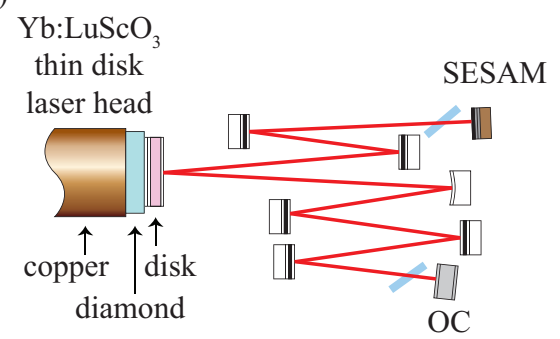

[||GTI 『 highly reflective mirror | YAG plate b)

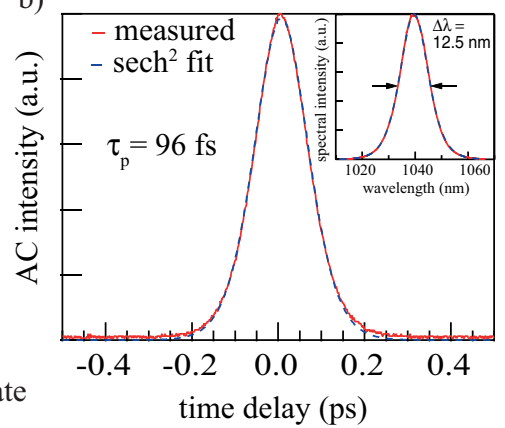

Fig. 2: Modelocked TDL with sub-100 fs pulse duration : a) Schematic of the laser cavity b) Autocorrelation trace and (inset) optical spectrum at $5.1 \mathrm{~W}$ output power

The experiment on CEO frequency detection was performed on a second laser setup (Fig.3) based on the gain material $\mathrm{Yb}: \mathrm{Lu}_{2} \mathrm{O}_{3}(12 \mathrm{~nm}$ FWHM emission bandwidth). The details of the experimental setup are presented in ref. [9]. We obtained pulses as short as $142 \mathrm{fs}$ at an average power of $7 \mathrm{~W}$ and an optical-to-optical efficiency of $15 \%$. The repetition rate was $64 \mathrm{MHz} .65 \mathrm{~mW}$ of the output power were enough to generate a stable octave-spanning supercontinuum (SC) in a 1-m 
long, highly nonlinear photonic crystal fiber. The SC was launched into an $f$-to- $2 f$ interferometer [10] for CEO beat detection. The observed CEO beats were tunable with the pump current. This mechanism will be used for electronics stabilization of the system to an external reference. It is worth emphasizing that CEO detection was possible despite the strongly multimode pumping scheme of TDLs, usually associated with a high noise level.
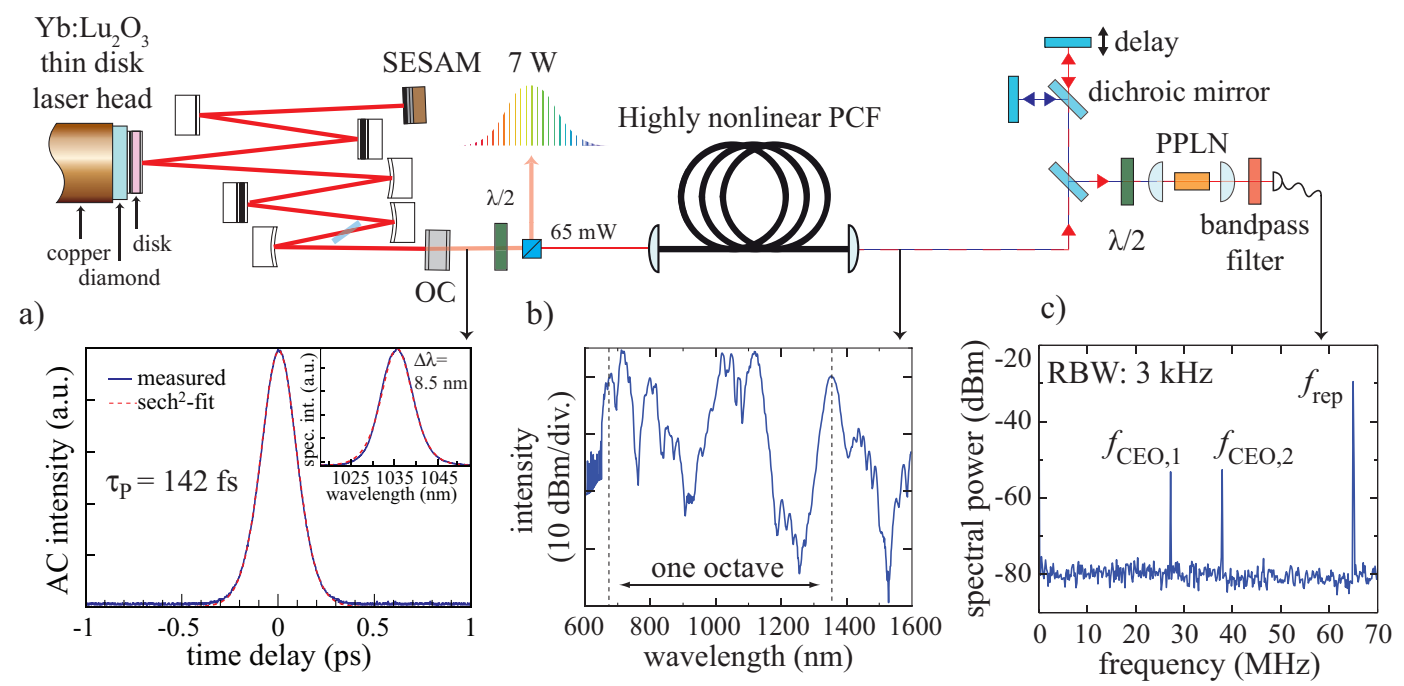

Fig. 3: Experimental setup for the CEO frequency detection of the $\mathrm{Yb}: \mathrm{Lu}_{2} \mathrm{O}_{3}$ TDL a) schematic of the TDL delivering $7 \mathrm{~W}$ of average power and $142 \mathrm{fs}$ pulses b) $1 \%$ of the output of the laser was launched into a highly nonlinear PCF, resulting in an octave spanning coherent super continuum c) $f$-to- $2 f$ interferometer for CEO frequency detection and observed CEO beat frequencies.

These proof-of-principle results confirm that TDLs are excellent candidates for driving high field physics experiments directly from table-top oscillators. In the near future we expect stabilized multi$100 \mathrm{~W}$ level oscillators with sub-100 fs pulse duration.

[1] A. Giesen, et al., "Scalable Concept for Diode-Pumped High-Power Solid-State Lasers," Appl. Phys. B 58, 365-372 (1994).

[2] U. Keller, et al., "Semiconductor saturable absorber mirrors (SESAMs) for femtosecond to nanosecond pulse generation in solid-state lasers," IEEE J. Sel. Top. Quantum Electron. 2, 435-453 (1996).

[3] C. R. E. Baer, et al., "Femtosecond thin disk laser with $141 \mathrm{~W}$ of average power," Optics Letters 35, 2302-2304 (2010).

[4] D. Bauer, et al., "Mode-locked Yb:YAG thin-disk oscillator with $41 \mathrm{uJ}$ pulse energy at 145 W average infrared power and high power frequency conversion," Optics Express 20, 96989704 (2012).

[5] T. Südmeyer, et al., "Femtosecond laser oscillators for high-field science," Nature Photonics 2, 599-604 (2008).

[6] T. Südmeyer, et al., "High-power ultrafast thin disk laser oscillators and their potential for sub-100-femtosecond pulse generation," Appl. Phys. B 97, 281-295 (2009).

[7] C. J. Saraceno, et al., "Sub-100 femtosecond pulses from a SESAM modelocked thin disk laser," Applied Physics B-Lasers and Optics 106, 559-562 (2012).

[8] C. J. Saraceno, et al., "SESAMs for high-power femtosecond modelocking: power scaling of an $\mathrm{Yb}$ :LuScO3 thin disk laser to $23 \mathrm{~W}$ and 235 fs," Optics Express 19 (2011).

[9] C. J. Saraceno, et al., "Self-referenceable frequency comb from an ultrafast thin disk laser," Optics Express 20 (2012).

[10] H. R. Telle, et al., "Carrier-envelope offset phase control: A novel concept for absolute optical frequency measurement and ultrashort pulse generation," Appl. Phys. B 69, 327-332 (1999). 